\title{
A BOUNDARY-INTEGRAL METHOD APPLIED TO WATER CONING IN OIL RESERVOIRS
}

\author{
S. K. LUCAS ${ }^{1}$, J. R. BLAKE ${ }^{2}$ and A. KUCERA ${ }^{3}$
}

(Received 1 April 1989; revised 13 March 1990)

\begin{abstract}
In oil reservoirs, the less-dense oil often lies over a layer of water. When pumping begins, the oil-water interface rises near the well, due to the suction pressures associated with the well. A boundary-integral formulation is used to predict the steady interface shape, when the oil well is approximated by a series of sources and sinks or a line sink, to simulate the actual geometry of the oil well. It is found that there is a critical pumping rate, above which the water enters the oil well. The critical interface shape is a cusp. Efforts to suppress the cone by using source/sink combinations are presented.
\end{abstract}

\section{Introduction}

An oil reservoir consists of a porous rock, like sandstone or limestone, in which oil is trapped in the interstices with some form of impermeable layer above, to keep it trapped (Muskat [14], Bear [2]). Water is often found in these reservoirs, and since it is more dense, it will lie in a layer below that of the oil, as shown in Figure 1(a). All fluid in the reservoir will be under a natural pressure due to the rock above. The temperature of the fluid will be in equilibrium with the rock. It is this natural pressure that, when a well is drilled, forces some of the oil to the surface, and is known as 'primary oil recovery'.

Pumping out of oil from the reservoir generates a suction pressure that acts on both the oil and the water, causing the oil-water interface to rise. To counterbalance this, we have gravity forces due to fluid density differences

\footnotetext{
${ }^{1}$ Department of Mechanical Engineering, University of Sydney, Sydney, N.S.W. 2006.

${ }^{2}$ School of Mathematics and Statistics, University of Birmingham, Birmingham B15 2TT U.K.

${ }^{3}$ Department of Mathematics, Australian Defence Force Academy, Campbell, A.C.T. 2600.

(C) Copyright Australian Mathematical Society 1991, Serial-fee code 0334-2700/91
} 


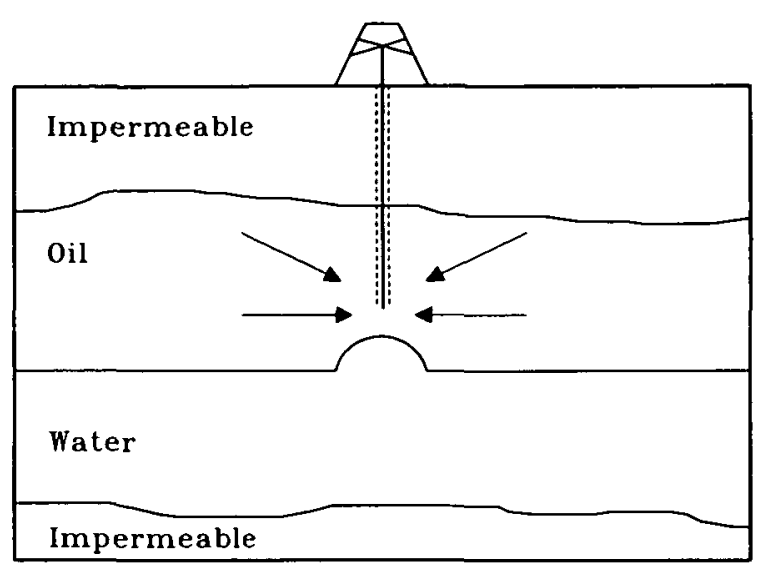

FIGURE 1(a). Water coning in an oil reservoir.

pulling the water down. If the well is pumping out oil at a constant rate, and if the pressure forces acting on the water are in equilibrium with the gravity forces, the oil-water interface will reach a stable shape below the well. If well forces are greater, the interface will reach up into the well after a finite time, and water will become a substantial portion of the well's production, which is not desirable. We can define the critical pumping rate as that such that a greater rate causes water to enter the well. At this rate, we would obtain a stable shape for the oil-water interface, but it is at a position about to break through to the well. This rising of the water layer towards the oil well, and sometimes breaking through and producing water, is known as 'water coning' in an oil reservoir. The objective of this paper is to determine the steady-state shape of this water cone, based upon a pumping rate or downhole pressure being specified. This is an extension of the earlier work by Blake and Kucera [4].

The motion of an interface between two immiscible or density-stratified fluids, where one is being withdrawn, is not a topic restricted to oil reservoirs. A similar phenomenon is often found in groundwater hydrology at the interface between fresh and salty water, as in coastal aquifers, where fresh water is pumped out. Another example, removed from flow in a porous medium, is a fresh-water reservoir, which can become stratified during long hot summers, where it is preferred to remove the more saline layers.

An extensive literature has developed on the analogous inviscid potentialflow problem of a sink near a free surface (see e.g. Craya [7], Tuck and Vanden-Broeck [16], Hocking [10, 11], Collings [6]). In that work, the limiting shape for the free surface is a cusp. 
Fluid movement in porous media has been analysed extensively by models with a modified Darcy's law, using relative permeabilities and saturations. These models, however, of ten suffer instabilities near the well itself, requiring restricted time steps as in MacDonald [13], or application of boundary conditions at some distance from the well [5]. This is due mainly to the vastly increasing flow rate of fluid as it approaches the sink, or well.

Muskat [14] was the first to suggest that Darcy's law for single-phase flows could be applied explicitly to each phase in a multiphase flow, which has subsequently become one of the major assumptions in oil-reservoir models. It must be noted that Darcy's law is only valid on the macroscopic level. At the microscopic level, instead of working with an overall permeability on the rock, we would have to get into the actual flow between interstices, and capillary forces would become far more important. In more realistic oilreservoir simulation models, a modified version of Darcy's law is used, which incorporates the idea of a relative permeability which is a non-linear function of the relative saturation of oil and water [8]. Thus, we emphasise that our water-coning model is on a macroscopic level, where pressure gradients and permeabilities are the dominant factors.

Another point of importance is the actual transition zone that exists between two fluids flowing in a porous medium. In many cases, this transition zone is narrow relative to the dimensions of the regions occupied by the fluids. In these cases, we can assume a sharp interface between fluids. This approximation, which is used in this project, has often been successfully used in oil-recovery studies (Hinch [9]), and is also valid for fresh-salt water transitions in coastal aquifers (Bear and Dagan [3]).

In two dimensions, sink-like flows near the interface between two immiscible fluids in a porous medium have been studied using hodograph techniques. Bear and Dagan [3] showed that at the critical flow rate, the interface forms a 'cusp' coming up to just below the sink. Throughout this paper, the word cusp will be used in the mathematical sense, as a singular point of a curve where the limits of tangents approaching from either side coincide. This shape is the limiting shape of the interface; any higher flow rates will lead to a breakthrough of the water into the well.

It should also be noted that most mathematical models (including our own) produce apparently-stable water-cone curves that, realistically, are not strictly stable. As oil is removed from the system, since oil reservoirs are only finite in size, changes both to underground pressure and the quantity of oil will alter our model. For example, as the oil is pumped out, the water-oil interface base level will rise, increasing the chance of water breakthrough, as the water cone itself will rise higher towards the well. Indeed, water is often pumped into the reservoir to replace the oil that is pumped out. This is one aspect of what is known as secondary oil recovery. 


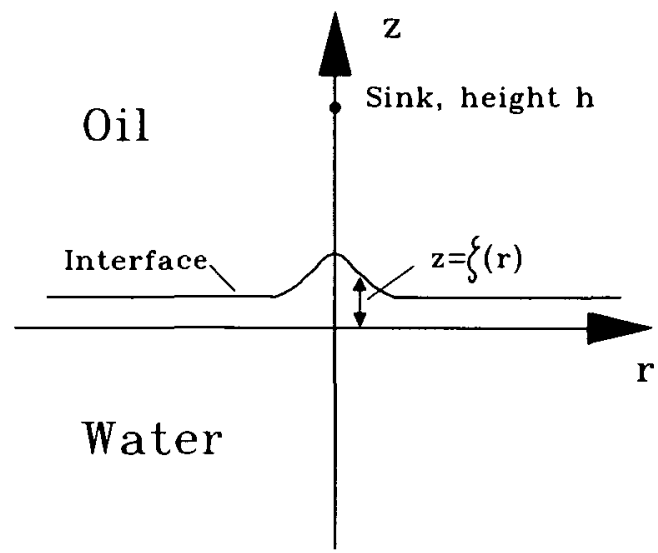

Figure l(b). Geometry of the model.

The other factor that also needs to be acknowledged is that the 'steady interface strategy' leading to a maximum pumping rate may not be the optimal strategy for maximising oil production. It may be desirable to pump at rates above this maximum pumping rate until the water cone reaches the well, and then to alter the pumping rate (see e.g. Ewing [6]). However we shall not concern ourselves with this problem in this paper, but shall instead concentrate solely on the steady-state problem.

\section{Equations}

To simplify the problem, we shall consider an homogeneous medium of constant permeability $k$ occupying all space. Figure 1(b) shows the basic geometry of the model. Furthermore, we are assuming that the fluids are immiscible and that, apart from the interface, all pore spaces are filled with one of the two fluids. Under these simplifying conditions, Darcy's law can then be applied independently to each region, assuming a constant permeability throughout, but allowing for different viscosities and densities.

We shall assume that the upper fluid has density $\rho_{1}$ and viscosity $\mu_{1}$, and the lower fluid has density $\rho_{2}\left(>\rho_{1}\right)$ and viscosity $\mu_{2}$. We have the fluids separated by an interface $z=\zeta(r, t)$. Darcy's law applied to both fluids yields

$$
\mathbf{u}^{(i)}=-\left(k / \mu_{i}\right) \nabla \hat{p}^{(i)} \text { for } i=1,2,
$$

where

$$
\hat{p}^{(i)}=p^{(i)}+\rho_{i} g z
$$

is the modified pressure, with $\rho_{2}>\rho_{1} \cdot \mathbf{u}^{(i)}$ is the velocity, $\mu_{i}$ the viscosity 
and $\rho_{i}$ is the density. $i=1$ corresponds to the upper fluid (oil) while $i=2$ corresponds to the lower fluid (water). The incompressibility condition in both fluids gives

$$
\nabla \cdot \mathbf{u}^{(i)}=0 .
$$

Taking (2.3) with (2.1) gives Laplace's equation for the modified pressures as

$$
\nabla^{2} \hat{p}^{(l)}=0
$$

with boundary conditions that $\mathbf{u}^{(i)}$ and $\hat{p}^{(i)}$ tend to zero at infinity. The dynamic boundary condition is that

$$
p^{(1)}=p^{(2)} \text { on } z=\zeta(r, t),
$$

and we also have the material boundary condition for particles on the interface, which in cylindrical polar co-ordinates is

$$
\frac{\partial \zeta}{\partial t}+u \frac{\partial \zeta}{\partial r}-v=0 \quad \text { on } z=\zeta(r, t)
$$

where $u$ is the radial $(r)$ velocity, and $v$ is the axial $(z)$ velocity.

The final required specification is that of the sink strength. Here the equations for point and line sinks will be developed (in Section 5, the arrangement for the constant downhole pressure model will be examined, which is slightly different from the ensuing analysis). For a single sink of volume flow rate $m$, modelled by a point sink and using the convention that $m \geq 0$, we have the expression for $p^{*}$, the suction pressure in an infinite porous medium, as

$$
p^{*}=\frac{-m \mu_{1}}{4 \pi k \sqrt{(z-h)^{2}+r^{2}}},
$$

where the sink is in the upper fluid at height $z=h$. If we were considering the general time-dependent problem, we would need to specify an initial shape, but since we are only considering a steady-state solution here, we do not require it. Indeed, the essential unknown quantity in this problem is the interface shape, which we shall see is only dependent on one dimensionless parameter, a 'suction or pump parameter' $F$. In addition, we have $\hat{p}^{(2)}=0$, since the steady-state problem requires no flow in the lower region. Thus, dropping the superscript for pressure in the upper fluid, we need to solve for pressure $\hat{p}$ given by

$$
\hat{p}=p^{*}+p^{\prime},
$$

where $p^{\prime}$ is the pressure variation from that due to a sink in an infinite fluid $\left(p^{*}\right)$. The dynamic boundary condition (2.5) becomes, using (2.2),

$$
g \zeta\left(\rho_{2}-\rho_{1}\right)+\hat{p}=0 \quad \text { on } z=\zeta(r, t) .
$$


If we scale all lengths with respect to $h$, and pressure with respect to $m \mu_{1} / k h$, we get the dimensionless form of the dynamic boundary condition from (2.9) as

$$
\tilde{\zeta}+F \tilde{p}=0 \quad \text { on } z=\zeta(r, t),
$$

where $\tilde{\zeta}, \tilde{p}$ are dimensionless height and pressure respectively, and

$$
F=\frac{m \mu_{1}}{k h^{2}\left(\rho_{2}-\rho_{1}\right) g}
$$

is the only dimensionless parameter, which represents the balance between the suction force of the sink and the gravitational restoring force of the denser fluid. Finally, of importance is the dimensionless suction pressure, which from (2.7) becomes

$$
p^{*}=\frac{-1}{4 \pi \sqrt{(z-1)^{2}+r^{2}}},
$$

where $z, r$ are now both dimensionless lengths.

\section{Boundary integral development}

We have, for a smooth function $\phi$ that satisfies Laplace's equation in a domain $\Omega$ with a piecewise smooth surface $S$, Green's integral formula (Blake, Taib and Doherty [5], Blake and Kucera [4]) as

$$
c \phi(p)+\int_{S} \phi(q) \frac{\partial G}{\partial n} d S=\int_{S} \frac{\partial \phi(q)}{\partial n} G d S,
$$

where $p \in \Omega+S, q \in S$, with $\partial / \partial n$ being the normal derivative outward from $S$,

$$
c=\left\{\begin{array}{ll}
1, & p \in \Omega \\
\frac{1}{2}, & p \in S
\end{array} \quad \text { and } \quad G=\frac{1}{4 \pi|p-q|} .\right.
$$

For our problem, we can rewrite (3.1) as

$$
c p(a)=p^{*}+\int_{S}\left(\frac{\partial p(b)}{\partial n} G-p(b) \frac{\partial G}{\partial n}\right) d S,
$$

where $a \in \Omega+S$ and $b \in S$. Since we are considering a steady-state problem, there is no motion normal to the interface, therefore $\partial p / \partial n=0$. If we then multiply (3.3) throughout by $(-F)$, and then apply the dynamic boundary condition (2.10), we obtain the integral representation for the interface shape as

$$
c \zeta=-F p^{*}-\int_{S} \zeta \frac{\partial G}{\partial n} d S,
$$


where $S$ is the surface $z-\zeta(r)=0$ for $r: 0 \rightarrow \infty$. Our domain $\Omega$ is here the upper (oil) fluid, so the outward normal will be down into the lower liquid.

Using cylindrical polar co-ordinates with $a=\left(r_{0}, 0, z_{0}\right), b=(r, \theta, z)$, then

$$
\begin{aligned}
G & =\frac{1}{4 \pi \sqrt{\left(r \cos \theta-r_{0} \cos 0\right)^{2}+\left(r \sin \theta-r_{0} \sin 0\right)^{2}+\left(z-z_{0}\right)^{2}}} \\
& =\frac{1}{4 \pi \sqrt{\left(r+r_{0}\right)^{2}+\left(z-z_{0}\right)^{2}-4 r r_{0} \cos ^{2} \theta / 2}} .
\end{aligned}
$$

Our surface is

$$
F(r, \theta, z)=z-\zeta(r),
$$

so that $F(r, \theta, z)=0$, and the unit normal (downwards) is

$$
\begin{aligned}
\mathbf{n} & =\frac{-\nabla F}{|\nabla F|} \\
& =\left(\frac{\partial \zeta}{\partial r}, 0-1\right) / \sqrt{\left(\frac{\partial \zeta}{\partial r}\right)^{2}+1} .
\end{aligned}
$$

After extensive manipulation we may express

$$
\begin{aligned}
\int_{S} \zeta \frac{\partial G}{\partial n} d S=\int_{0}^{\infty} \frac{\zeta r}{\pi\left[\left(r+r_{0}\right)^{2}+\left(\zeta(r)-\zeta\left(r_{0}\right)\right)^{2}\right]^{3 / 2}} \\
\quad \times\left[\frac{E(\mathbf{k})}{1-\mathbf{k}^{2}}\left\{\frac{2 r_{0} \frac{\partial \zeta}{\partial r}}{\mathbf{k}^{2}}-\left(r+r_{0}\right) \frac{\partial \zeta}{\partial r}+\left(\zeta(r)-\zeta\left(r_{0}\right)\right)\right\}-\frac{\left.2 r_{0} K(\mathbf{k}) \frac{\partial \zeta}{\partial r}\right]}{\mathbf{k}^{2}}\right] d r,
\end{aligned}
$$

where $K$ and $E$ are complete elliptic integrals of the 1st and 2nd kinds, (Abramowitz and Stegun [1]) and

$$
\mathbf{k}^{2}=\frac{4 r r_{0}}{\left(r+r_{0}\right)^{2}+\left(\zeta(r)-\zeta\left(r_{0}\right)\right)^{2}} .
$$

Now since we are interested in points on the boundary $z=\zeta(r)$, which we actually want to find, we take $c=\frac{1}{2}$. Changing the variables so that the given point is at radius $r$ and the dummy variable is $\lambda$, we then have

$$
\frac{1}{2} \zeta(r)=-F p^{*}-\int_{0}^{\infty} \lambda \zeta(\lambda) \mathbf{K}(r, \lambda) d \lambda,
$$


where

$$
\begin{aligned}
\mathbf{K}(r, \lambda)= & \frac{1}{\pi\left[(\lambda+r)^{2}+(\zeta(\lambda)-\zeta(r))^{2}\right]^{3 / 2}} \\
& \times\left[\frac{E(\mathbf{k})}{1-\mathbf{k}^{2}}\left\{\frac{2 r \frac{\partial \zeta}{\partial \lambda}}{\mathbf{k}^{2}}-(\lambda+r) \frac{\partial \zeta}{\partial \lambda}+(\zeta(\lambda)-\zeta(r))\right\}-\frac{2 r \frac{\partial \zeta}{\partial \lambda} K(\mathbf{k})}{\mathbf{k}^{2}}\right] \\
& \mathbf{k}^{2}=\frac{4 r \lambda}{(\lambda+r)^{2}+(\zeta(\lambda)-\zeta(r))^{2}}
\end{aligned}
$$

and

$$
p^{*}=\frac{-1}{4 \pi \sqrt{(\zeta-1)^{2}+r^{2}}} .
$$

The problem is now specified as a nonlinear Fredholm integral equation of the second kind for the unknown interface shape $z=\zeta(r)$, in which the suction pressure $p^{*}$ is specified by the parameter $F$.

Equation (3.10) can be solved using a standard fixed-point type iteration technique, starting with the 1st order small-parameter approximation (Blake and Kucera [4]) as an initial approximation $\zeta^{0}(r)$, where

$$
\zeta_{0}(r)=\frac{F}{2 \pi\left(r^{2}+1\right)^{\frac{1}{2}}}
$$

Our method is to substitute $\zeta^{0}(r)$ into the right-hand side of (3.10), and so find the next function $\zeta^{1}(r)$ as the left-hand side. This process will generate a sequence of functions $\left\{\zeta^{n}(r)\right\}_{n=1}^{\infty}$, that will converge pointwise to $\zeta(r)$, our final interface solution. Table 1 shows that few iterations are required for this convergence to be achieved.

The integration in (3.10) was performed using QUADPACK (a numerical integration package - see Piessens et al [15]). Investigation of the kernel function $K$ from (3.11) shows a singularity when $\lambda=r$. Because of this, the integral was split into two parts: $0 \leq \lambda \leq r$ and $r \leq \lambda \leq \infty$, placing the singularity in the integral at the endpoints. These integrations were performed using DQAGS and DQAGI respectively. QUADPACK readily achieved the accuracy required for both integrals. $\zeta^{n}(r)$ was interpolated using a clamped cubic spline from 0 to $r_{\max }$ with end conditions being $\zeta^{\prime n}(0)=0$ (since it is only at the critical rate that a cusp occurs) and $\zeta^{\prime n}\left(r_{\max }\right)=\zeta^{\prime 0}\left(r_{\max }\right)$ where $\zeta^{\prime 0}(r)$ is taken from the 1st order small-parameter expansion above. Further, we take for $r>r_{\max }, \zeta^{n}(r) \equiv \zeta^{0}(r)$.

For the QUADPACK numeric integration package to work, a function defining the integrand is required as the integrand, rather than a set of points. 
TABLE 1. Iterations required for the various situations to converge.

\begin{tabular}{|c|c|c|c|c|c|c|c|c|c|c|}
\hline \multicolumn{2}{|c|}{ Point Sink } & \multicolumn{5}{|c|}{ A Point Sink and a Point Source } & \multicolumn{4}{|c|}{ Line Sink } \\
\hline$F$ & its & $F$ & $h 1$ & $F$ & $h 2$ & its & $F$ & $h 1$ & $h 2$ & its \\
\hline 0.2 & 2 & 2.2 & 1.0 & -0.2 & 1.0 & 11 & 2.0 & 1.0 & 1.0 & 11 \\
\hline 0.4 & 3 & 2.2 & 1.0 & -0.2 & 0.9 & 10 & 2.0 & 1.0 & 1.5 & 4 \\
\hline 0.6 & 3 & 2.2 & 1.0 & -0.2 & 0.8 & 8 & 2.0 & 1.0 & 2.0 & 4 \\
\hline 0.8 & 3 & 2.2 & 1.0 & -0.2 & 0.7 & 7 & 2.0 & 1.0 & 2.5 & 4 \\
\hline 1.0 & 4 & 2.2 & 1.0 & -0.2 & 0.6 & 6 & 2.0 & 1.0 & 3.0 & 4 \\
\hline 1.2 & 4 & 2.2 & 1.0 & -0.2 & 0.6 & 6 & 2.0 & 1.0 & 3.5 & 4 \\
\hline 1.4 & 4 & 2.2 & 1.0 & -0.2 & 0.4 & 10 & 0.5 & 1.0 & 2.0 & 3 \\
\hline 1.6 & 5 & 2.2 & 1.0 & -0.2 & 0.8 & 10 & 1.0 & 1.0 & 2.0 & 3 \\
\hline 1.8 & 5 & 2.2 & 1.0 & -0.3 & 0.7 & 8 & 1.5 & 1.0 & 2.0 & 4 \\
\hline 2.0 & 11 & 2.2 & 1.0 & -0.3 & 0.6 & 6 & 2.0 & 1.0 & 2.0 & 4 \\
\hline-1.0 & 3 & 2.5 & 1.0 & -0.3 & 0.5 & 9 & 2.5 & 1.0 & 2.0 & 5 \\
\hline-3.0 & 4 & 2.5 & 1.0 & -0.3 & 0.4 & 14 & 3.0 & 1.0 & 2.0 & 7 \\
\hline-5.0 & 4 & & & & & & 3.5 & 1.0 & 2.0 & 7 \\
\hline-7.0 & 5 & & & & & & & & & \\
\hline-9.0 & 6 & & & & & & & & & \\
\hline
\end{tabular}

We used a cubic spline interpolation method to produce a smooth differentiable piecewise polynomial $S$, where cubic polynomials are found between successive points. A cubic spline gives enough undetermined constants so that the curve is not only continuously differentiable on the interval, but has a continuous second derivative as well. Linear interpolation was tried between points, but was found to be unacceptable. The discontinuities in the derivative of $\zeta$ caused QUADPACK to be much slower, and the convergence of the iteration was not as successful for the curves produced close to critical $F$. Since this is where most interest is, it was decided that linear interpolation was too inaccurate for our problem. The cubic spline has the advantage 
of producing both a continuous curve and derivative, and gives a far superior level of accuracy.

However, there are problems with the above procedure. When $r=0$, the integral becomes split into the two parts: $0 \rightarrow 0$ and $0 \rightarrow \infty$ with the singularity at 0 . On the UNIVAC 1100 , this was not a problem since the bitsize is large enough, while the accuracy is not enough to ensure exact values of 0 . However, problems were introduced when the program was moved to an NEC APC IV. Errors of $0 / 0$ type were encountered on the identical code that worked on UNIVAC. An attempt was made to get around this problem when $r=0$ by making the integration range $0 \rightarrow \infty$, and assuming the $0 \rightarrow 0$ integral is zero. This allowed the program to run, but introduced different values for $\zeta(0)$. The errors are normally so small as to be invisible graphically. However, for the large values of negative $F$ (see Section 4), this error became large enough to cause a significant and highly visible spike at $r=0$, which was not valid. For $F=-9$, an error of $\sim 0.03$ (roughly $3 \%$ was found, which was clearly not supportable. Our way around this problem was to simplify the integration when $r=0$, which is on the axis of symmetry. Setting $r=0$ in (3.10) gives the expression

$$
c \zeta(0)=-F p^{*}-\int_{0}^{\infty} \lambda \zeta(\lambda) \mathbf{K}(0, \lambda) d \lambda,
$$

where

$$
\mathbf{K}(0, \lambda)=\frac{\zeta(\lambda)-\zeta(0)-\lambda \frac{\partial \zeta}{\partial \lambda}}{2\left[\lambda^{2}+(\zeta(\lambda)-\zeta(0))^{2}\right]^{3 / 2}} .
$$

This integrand is singular at the origin only, and when used gave identical results to those of the UNIVAC. This method was used throughout for axisymmetric problems.

\section{Point and line sinks}

(i) Point sink Figure 2(a) reproduces the results of Blake and Kucera [4], showing the interface shape and slope for $F=0.2,0.4, \ldots, 2.0$. The slope graph is useful in recognising cusping. The graphs were produced with a 100 point spline in the range $0 \rightarrow 5.0$, and then reproduced on the negative axis for aesthetic reasons. Table 1 shows the number of iterations required for convergence for each $F$. 100-point splines were used, since investigation of accuracy of various numbers of points showed this to be acceptable. Table 2 indicates how convergence varies away from under the sink.

Of most interest here is the critical pumping rate $F_{c}$, the maximum allowable rate of withdrawal that produces a stable cone beneath the well. For 
a

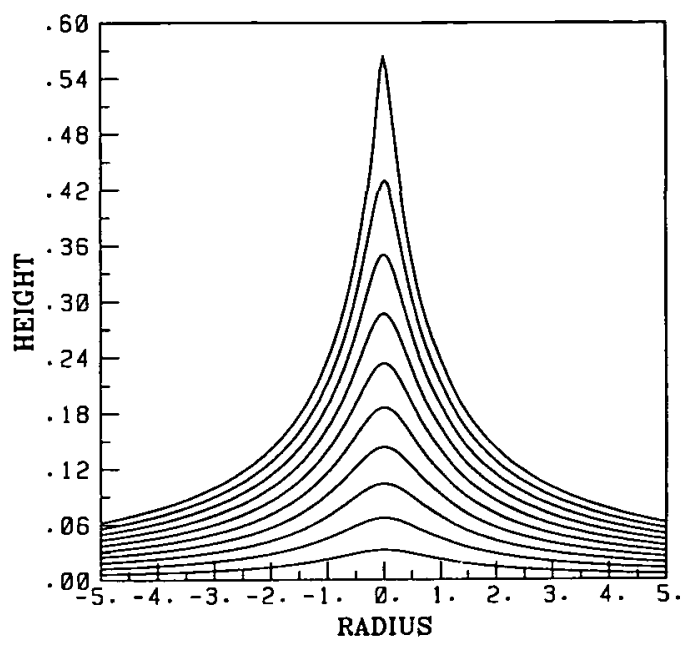

b

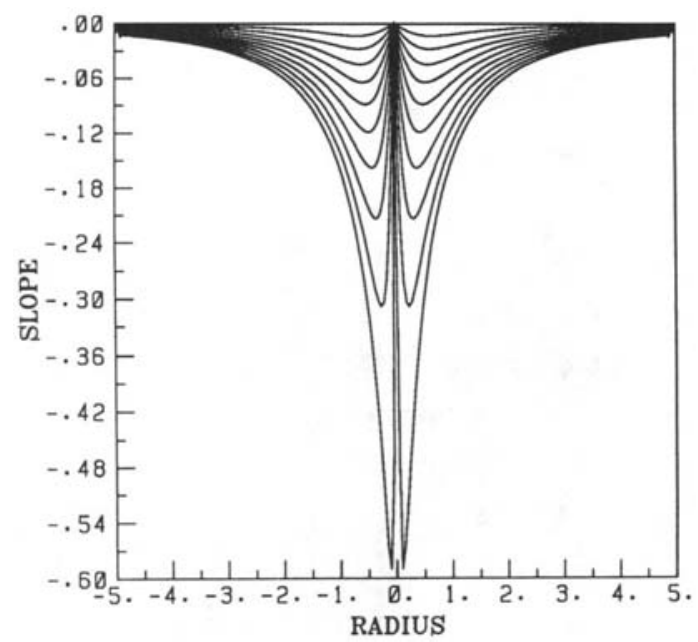

Figure 2. (a) Interface shape and (b) slope of interface for $F=0.2,0.4, \ldots, 2.0,100 \mathrm{pt}$ spline over range $0 \rightarrow 5$.

$F>F_{c}$, the cone is drawn into the well, and this breakthrough causes water production, which is undesirable. The value of $F_{c}$ has been found by a simple testing process to find at what maximum value of $F$ a converged cone shape is possible. Table 3 shows the values of $F_{c}$ determined to various accuracies, as well as the height of the interface directly under the sink. The values shown are the largest values of $F$, to the required accuracy, that give a stable cone. Note that when $F$ was too large, our model produced an iteration with $\zeta$ greater than our dimensionless point-sink height 1.0 when 
TABLE 2. Values of $\zeta$ on sucessive iterations; $100 \mathrm{pt}$ spline, range 5 .

\begin{tabular}{|c|c|c|c|c|c|c|}
\hline \multicolumn{4}{|c|}{$F=0.5$} & \multicolumn{3}{|c|}{$F=1.0$} \\
\hline$N$ & $\zeta(0)$ & $\zeta(1)$ & $\zeta(3)$ & $\zeta(0)$ & $\zeta(1)$ & $\zeta(3)$ \\
\hline 0 & 0.0796 & 0.0563 & 0.0252 & 0.1592 & 0.1125 & 0.0503 \\
\hline 1 & 0.0851 & 0.0572 & 0.0251 & 0.1838 & 0.1165 & 0.0502 \\
\hline 2 & 0.0853 & 0.0572 & 0.0251 & 0.1862 & 0.1162 & 0.0502 \\
\hline 3 & 0.0853 & 0.0572 & 0.0251 & 0.1863 & 0.1162 & 0.0502 \\
\hline 4 & 0.0853 & 0.0572 & 0.0251 & 0.1863 & 0.1162 & 0.0502 \\
\hline 5 & 0.0853 & 0.0572 & 0.0251 & 0.1863 & 0.1162 & 0.0502 \\
\hline 6 & 0.0853 & 0.0572 & 0.0251 & 0.1863 & 0.1162 & 0.0502 \\
\hline 7 & 0.0853 & 0.0572 & 0.0251 & 0.1863 & 0.1162 & 0.0502 \\
\hline 8 & 0.0853 & 0.0572 & 0.0251 & 0.1863 & 0.1162 & 0.0502 \\
\hline 9 & 0.0853 & 0.0572 & 0.0251 & 0.1863 & 0.1162 & 0.0502 \\
\hline 10 & 0.0853 & 0.0572 & 0.0251 & 0.1863 & 0.1162 & 0.0502 \\
\hline \multicolumn{4}{|c|}{$F=1.5$} & \multicolumn{3}{|c|}{$F=2.0$} \\
\hline$N$ & $\zeta(0)$ & $\zeta(1)$ & $\zeta(3)$ & $\zeta(0)$ & $\zeta(1)$ & $\zeta(3)$ \\
\hline 0 & 0.2387 & 0.1688 & 0.0755 & 0.3183 & 0.2251 & 0.1007 \\
\hline 1 & 0.3014 & 0.1779 & 0.0751 & 0.4453 & 0.2415 & 0.1000 \\
\hline 2 & 0.3154 & 0.1766 & 0.0751 & 0.5092 & 0.2380 & 0.0998 \\
\hline 3 & 0.3173 & 0.1765 & 0.0751 & 0.5424 & 0.2374 & 0.0998 \\
\hline 4 & 0.3173 & 0.1766 & 0.0751 & 0.5579 & 0.2375 & 0.0998 \\
\hline 5 & 0.3173 & 0.1766 & 0.0751 & 0.5635 & 0.2376 & 0.0998 \\
\hline 6 & 0.3173 & 0.1766 & 0.0751 & 0.5645 & 0.2376 & 0.0998 \\
\hline 7 & 0.3173 & 0.1766 & 0.0751 & 0.5640 & 0.2376 & 0.0998 \\
\hline 8 & 0.3173 & 0.1766 & 0.0751 & 0.5637 & 0.2376 & 0.0998 \\
\hline 9 & 0.3173 & 0.1766 & 0.0751 & 0.5636 & 0.2376 & 0.0998 \\
\hline 10 & 0.3173 & 0.1766 & 0.0751 & 0.5437 & 0.2376 & 0.0998 \\
\hline
\end{tabular}

$r$ was near zero, indicating fluid breakthrough. Also, getting any more than 3 decimal places would be difficult; $F=2.049$ had $\zeta(0)>1.0$ after 33 iterations, which took about five and a half hours on an NEC APC IV.

Observing the slope graph in Figure 2(b), we can also get some insight into the limiting shape of the interface. We can see how the maximum slope, as well as getting larger as $F$ increases, is at a point that is moving inwards towards $r=0$, under the sink. This indicates that the interface shape is approaching a cusp, a result also obtained by Bear and Dagan [3] using the 
TABLE 3. Critical Suction and $\zeta(0)$ to increasing accuracy.

\begin{tabular}{|c|l|c|c|}
\hline$d p$ accuracy & $F_{c}$ & $\zeta(0)$ & Iterations required \\
\hline 0 & 2 & 0.5637 & 11 \\
1 & 2.0 & 0.5637 & 11 \\
2 & 2.04 & 0.6247 & 15 \\
3 & 2.048 & 0.6540 & 18 \\
\hline
\end{tabular}

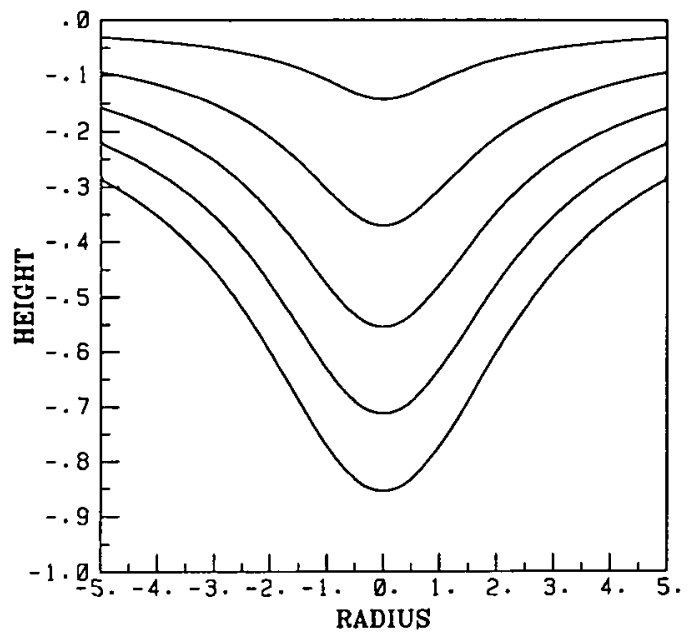

Figure 3. Interface shape for $F=-1,-3, \ldots,-9,100 \mathrm{pt}$ spline over range $0 \rightarrow 5$.

hodograph method. We should note that the hodograph method is only a 2-D method, and would not be applicable to general 3-D problems.

(ii) Point source Instead of a point sink which is used to model the removal of oil from the reservoir, by making $F$ negative we produce a point source which pumps liquid into the reservoir. This will have the effect of depressing the interface near the source, and will avoid the cusping effect which occurs for positive $F$, since distance from the source increases as $F$ increases (in a negative direction). Figure 3 shows shape and slope graphs for $F=$ $-1,-3, \ldots,-9$. We can see that the ratio of successive interface depths $(\zeta(0))$ gets smaller as we increase $F$, that is,

$$
\frac{\left.\zeta(0)\right|_{F=1}}{\left.\zeta(0)\right|_{F=3}}>\frac{\left.\zeta(0)\right|_{F=3}}{\left.\zeta(0)\right|_{F=5}}>\frac{\left.\zeta(0)\right|_{F=5}}{\left.\zeta(0)\right|_{F=7}} \text { etc, }
$$

which is expected when we consider the equations relating pressure to distance. Further discussion on this example may be found in Lucas [8]. 
(iii) Multiple sinks/sources When (3.10) is examined, we can see that the integrand has no dependence on $F$. In fact, the only place where $F$ and the height of the sink are found is in the portion $-F p^{*}$. Note that scaling of lengths was done so that height was taken as 1 . Since this is such a simple expression for sink strength and position, it is not difficult to extend this to multiple sinks and/or sources. Scaling in this case is done so that one of the sinks/sources is placed at 1 , and others can be scaled with respect to it. Therefore, for multiple sink/source case, (3.10) becomes

$$
\frac{1}{2} \zeta(r)=\left[\sum_{i=1}^{n} F_{i} p_{i}^{*}\right]-\int_{0}^{\infty} \lambda \zeta(\lambda) \mathbf{K}(r, \lambda) d \lambda,
$$

where there are $n$ sinks at height $h_{l}$, each sink has a sink strength of $F_{i}$, and

$$
p_{t}^{*}=\frac{-1}{4 \pi \sqrt{\left(\zeta-h_{\imath}\right)^{2}+r^{2}}} .
$$

The summation is to take into account the contribution to the pressure of each sink or source. Note that these multiple sinks/sources are such that they are placed at position $\left(0,0, h_{i}\right)$ in Cartesian coordinates, so that the axisymmetric nature of the problem is retained.

Figures 4(a) and (b) show two slightly different cases, having a smaller source directly under a sink. The reason for these choices is an attempt to suppress the water cone directly under the well. In Figure 4(a), we can see that the sum of $F$ 's is 2.0 , which is just under the critical $F$ for a single sink, and so having both sink and source at $h=1.0$ is possible. In an effort to extract oil at a greater than critical amount we may consider Figure 4(b), where the sum of $F$ 's is greater than the critical $F_{c}$; in other words, having both the source and sink at 1.0 is not going to give a stable interface. Indeed $F=-0.3$ at 0.9 also doesn't produce a stable cone for $F=2.5$ at height 1.0, but other examples of a stable cone are shown in Figure 4(b).

Note that in both Figures, we have stopped with the lower source at height 0.4. When the source is lower (e.g. at 0.3), the model breaks down. This is due to the fact that, as the source goes down between the humps produced by this configuration, its push outwards will be more significant than its effect downwards. This ballooning out will cause the interface near this low source to become many-valued in $r$. Our model as it stands is unable to cope with this extreme situation, and so breaks down.

(iv) Line sink The last extension to the axisymmetric model is to extend the point sink to a vertical line with a total suction $F$ over its length. This is an attempt to improve our model, since an oil well is more of a line of suction, rather than just a point. The line sink is the limiting case of having $n$ sinks of suction $F / n$ equally spaced along our finite vertical line segment, and then 
a
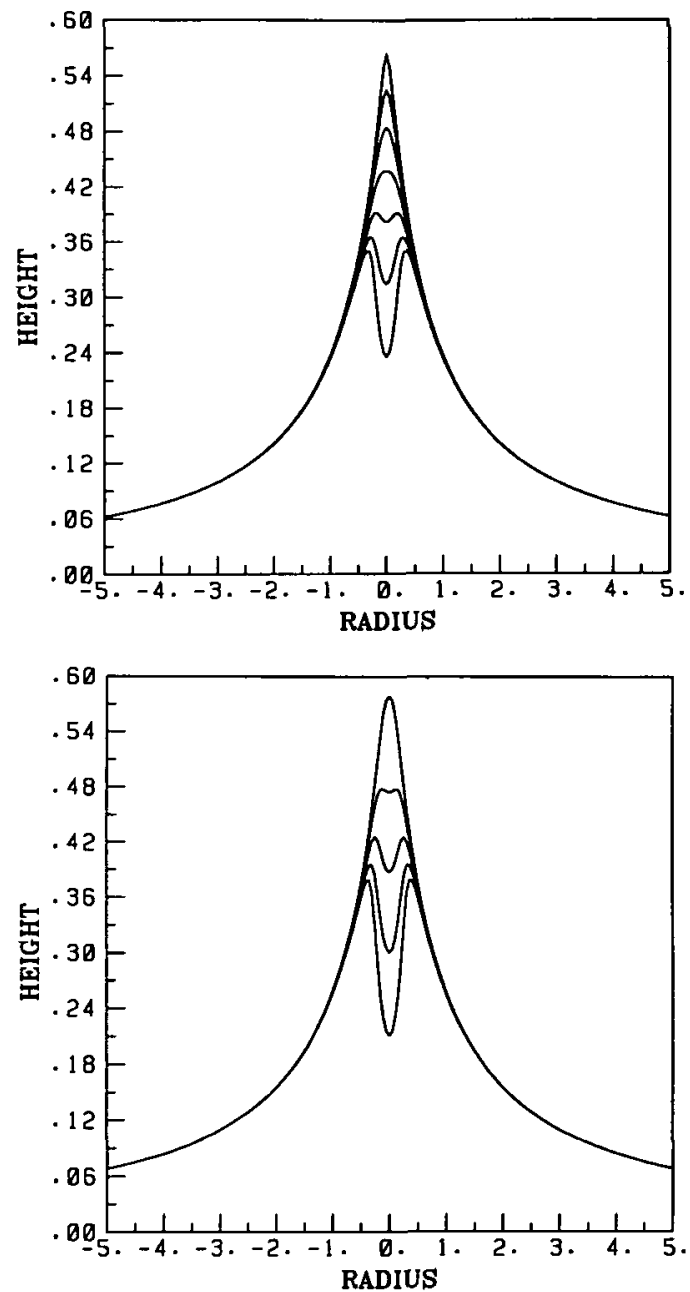

FIGURE 4. Interface shape for (a) $F=2.2$ at $h=1.0$ and $F=-0.2$ at $h=1.0,0.9$, $\ldots, 0.4$, and (b) $F=2.5$ at $h=1.0$ and $F=-0.3$ at $h=0.8,0.7, \ldots, 0.4,100 \mathrm{pt}$ spline over range $0 \rightarrow 5$.

taking the limit as $n \rightarrow \infty$. We use the endpoints of the line as $\left(0,0, h_{1}\right)$ and $\left(0,0, h_{2}\right)\left(h_{2}>h_{1}\right)$. Using the parametric representation of this line

$$
z=h_{1}+t\left(h_{2}-h_{1}\right)
$$

as $t: 0 \rightarrow 1$, then we have our basic equation

$$
c \zeta(r)=-F \int_{0}^{1} p^{*}(t) d t-\int_{0}^{\infty} \lambda \zeta(\lambda) K(r, \lambda) d \lambda,
$$


a
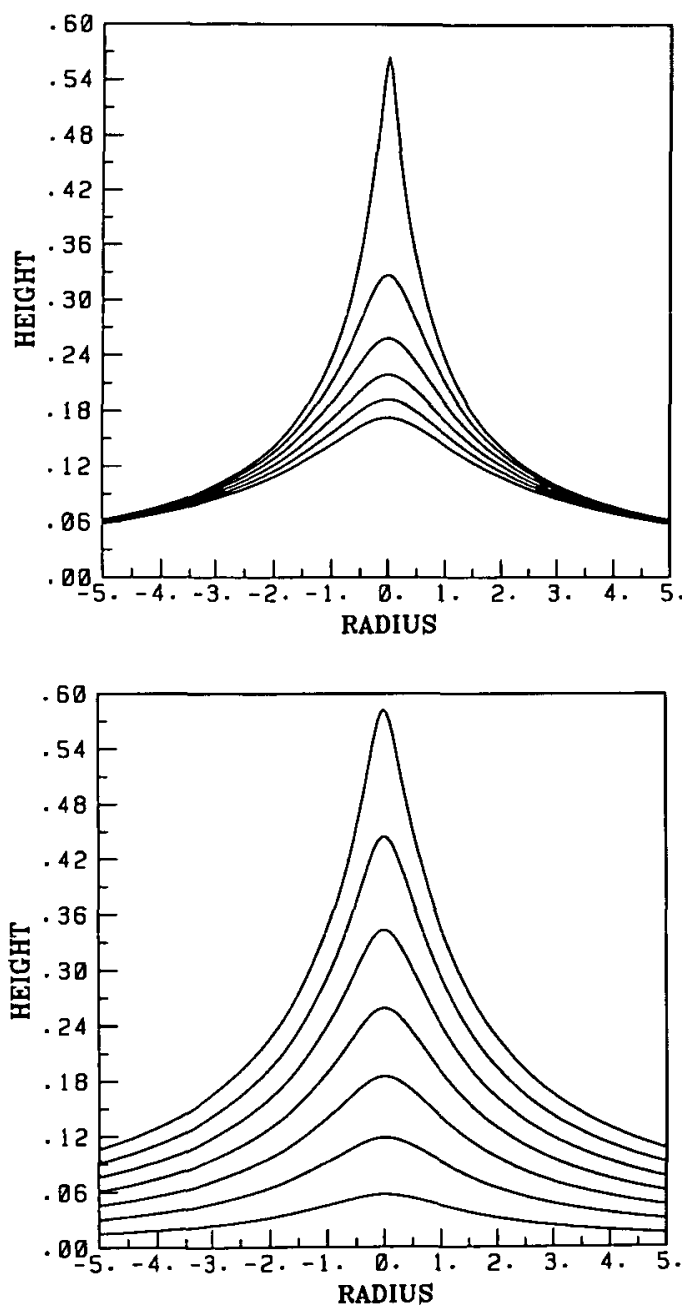

FIGURE 5. Interface shape for (a) $F=2.0$ over line sink from 1 to $1+n, n=$ $0.0,0.5, \ldots, 2.5$, and (b) $F=0.5,1.0, \ldots, 3.5$ over line sink from 1 to $2,100 \mathrm{pt}$ spline over range $0 \rightarrow 5$.

where

$$
p^{*}(t)=\frac{-1}{4 \pi \sqrt{\left(\zeta-\left(h_{1}+t\left(h_{2}-h_{1}\right)\right)\right)^{2}+r^{2}}} .
$$

For scaling considerations, taking $h_{1}=1$ and finding $h_{2}$ in an appropriate manner is a simple solution.

Two sets of runs for a line sink have been shown here. Figure 5(a) shows the effect of increasing the length of the line sink, while keeping the overall 
value of $F$ constant (at 2.0). The effect is as expected; the height is reduced as a greater proportion of the suction occurs further from the interface. Note also that for a line length of zero (the line degenerates into a point), we obtain identical results to those obtained using the point-sink program. Figure 5(b) shows results for a line sink of length one, with endpoints at heights 1 and 2 , with varying values of $F$. Naturally, $F$ values can go higher than for the point sink, but of interest is the fact that the curve is far smoother than for the point sink for values of $F$ leading to equivalent interface heights.

\section{Constant downhole-pressure formulation}

Previously, we have modelled our oil well by point sinks, point sources, or line sinks with a constant mass flow over them. In reality, an oil well is more like a cylinder upon which a suction (negative) pressure generates an oil flow towards the well. A better model, then, would be a cylinder of small radius, upon which some constant downhole pressure will operate. The downhole pressure is usually very much less (e.g. 10-100 atm less) than the local ambient pressure and will be represented as a negative quantity in the ensuing analysis. Additionally, the downhole pressure would not truly be constant, due to varying depth, but this change can be assumed to be negligible ccmpared to the ambient pressure (e.g. $1 \mathrm{~atm}$ in $100 \mathrm{~atm}$ ). Figure 6 shows this more realistic model. We will simulate a constant downhole pressure by a line sink at $r=(0,0, z)$, where $h \leq z \leq h+L$. This will give us, outside the pipe, the pressure due to the line sink as

$$
p^{*}=-\frac{\mu_{1}}{4 \pi k} \int_{h}^{h+L} \frac{m(\xi)}{\sqrt{(z-\xi)^{2}+r^{2}}} d \xi,
$$

where $m(\xi)$ is the (positive) flow rate per unit length into the pipe at position $h \leq \xi \leq h+L$ along the pipe. We shall specify the pipe radius as $r_{0}$, and assume $r_{0} \ll L$.

We defined previously

$$
\hat{p}=p^{*}+p^{\prime}
$$

where $p^{\prime}$ is the variation in pressure from that caused by the sink distribution in an infinite fluid. Since $r_{0} / h$ is small, we can neglect $p^{\prime}$ on the pipe to arrive at the approximation that $\hat{p}=p^{*}$. This approximation has the effect of simplifying the problem to two decoupled integral equations (5.3 and 5.8). This applied to (5.1) will give us, with $p_{d h}$ being the (constant and negative) 


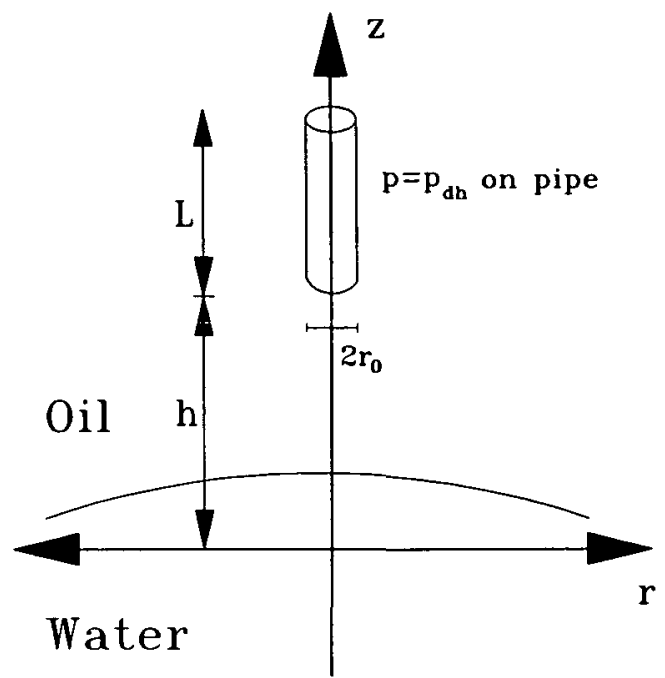

FIGURE 6. Geometry of the constant-downhole-pressure model.

downhole pressure,

$$
p_{d h}=-\frac{\mu_{1}}{4 \pi k} \int_{h}^{h+L} \frac{m(\xi)}{\sqrt{(z-\xi)^{2}+r_{0}^{2}}} d \xi \text { for } h \leq z \leq h+L .
$$

Equation (5.3) implies that $m(\xi)$ will not be constant over the entire length of the cylinder, as has been the basis of previous work. Since downhole pressure, $p_{d h}$, is the parameter that will cause interface height variation, rather than the mass flow $m$, we shall scale length with respect to $h$ as before, scale mass flow $m$ per unit length with respect to $-k p_{d h} / \mu_{1}$ (negative since $p_{d h}$ is negative, and we want to keep $m$ positive), and scale pressure with respect to $p_{d h}$.

This scaling will alter (2.9) to

$$
\tilde{\zeta}+\frac{p_{d h}}{g h\left(\rho_{2}-\rho_{1}\right)} \tilde{p}=0 \quad \text { on } z=\tilde{\zeta}(r),
$$

which, on defining $F^{\prime}=-p_{d h} /\left(g h\left(\rho_{2}-\rho_{1}\right)\right)$, a positive quantity since $p_{d h}<$ 0 , leads to

$$
\tilde{\zeta}-F^{\prime} \tilde{p}=0 .
$$

Equation (5.1) will become

$$
\tilde{p}^{*}=\frac{1}{4 \pi} \int_{1}^{1+\tilde{L}} \frac{\tilde{m}(\xi)}{\sqrt{(z-\xi)^{2}+\tilde{r}^{2}}} d \xi,
$$


and (5.3) will become

$$
\frac{1}{4 \pi} \int_{1}^{1+\widetilde{L}} \frac{\tilde{m}(\xi)}{\sqrt{(z-\xi)^{2}+\tilde{r}_{0}^{2}}} d \xi=1 \text { for } 1 \leq z \leq 1+\widetilde{L} .
$$

Using this altered scaling, and dropping the tildes, (3.10) will become

$$
\frac{1}{2} \zeta(r)=\frac{F^{\prime}}{4 \pi} \int_{1}^{1+L} \frac{m(\xi)}{\sqrt{(\zeta-\xi)^{2}+r^{2}}} d \xi-\int_{0}^{\infty} \lambda \zeta(\lambda) \mathbf{K}(r, \lambda) d \lambda .
$$

Comparison with (3.10) indicates that an increasingly positive value of $F^{\prime}$ will give an increasingly higher water-oil interface. The mass-flow integral can be calculated from (5.7).

This final requirement to solve (5.8) numerically is to solve (5.7) for $m$. We discretise $m$ into $n$ intervals of length $h=L / n$ and solve (5.7) for the mass flow $m_{i}$ at points $z_{i}=1+i h$ for $i=0,1, \ldots, n$. We evaluate $m(\xi)$ as constant between our $z_{i}$ 's, and since now there are only $n$ unknowns ( $\left.m_{i} ; i=1,2, \ldots, n\right)$, we can form a set of $n$ equations in $n$ unknowns by evaluating at the points $z=\left(z_{k-1}+z_{k}\right) / 2$, for $k=1,2, \ldots, n$. The $i$ th integral of the $j$ th equation $(j=1,2, \ldots, n ; i=1,2, \ldots, n)$ will be

$$
\begin{aligned}
\int_{z_{1-1}}^{z_{i}} \frac{m(\xi)}{\sqrt{(a-\xi)^{2}+r_{0}^{2}}} d \xi & =m_{i} \int_{z_{i-1}}^{z_{i}} \frac{d \xi}{\sqrt{(a-\xi)^{2}+r_{0}^{2}}} \\
& =m_{i} \ln \left[\frac{a-z_{i-1}+\sqrt{\left(a-z_{i-1}\right)^{2}+r_{0}^{2}}}{a-z_{i}+\sqrt{\left(a-z_{i}\right)^{2}+r_{0}^{2}}}\right],
\end{aligned}
$$

where

$$
a=\frac{z_{j-1}+z_{j}}{2}
$$

Thus, we shall solve

$$
A \mathbf{m}=\mathbf{b}
$$

where

$$
\mathbf{m}=\left(m_{0}, m_{1}, \ldots, m_{n}\right)^{\top}, \quad \mathbf{b}=(4 \pi, 4 \pi, \ldots, 4 \pi)^{\top},
$$

and $A=\left(a_{j i}\right)$, where

$$
a_{j i}=\ln \left[\frac{a-z_{i-1}+\sqrt{\left(a-z_{\imath-1}\right)^{2}+r_{0}^{2}}}{z-z_{i}+\sqrt{\left(a-z_{\imath}\right)^{2}+r_{0}^{2}}}\right] .
$$

Equation (5.8) can then be used to check the left-hand side of (5.7).

In the calculations, we use $L=1, r_{0}=0.005$ and $n=100$, which yields an almost constant value of $m$ over the pipe, except near the ends where $m$ 
increases rapidly, as would be expected from slender-body theory. A more detailed discussion of this solution technique and the errors involved can be found in Lucas [12].

\section{Constant downhole-pressure solution}

We solve

$$
\frac{1}{2} \zeta(r)=\frac{F^{\prime}}{4 \pi} \int_{1}^{1+L} \frac{m(\xi)}{\sqrt{(\zeta-\xi)^{2}+r^{2}}} d \xi-\int_{0}^{\infty} \lambda \zeta(\lambda) \mathbf{K}(r, \lambda) d \lambda
$$

as before, using

$$
\int_{z_{l-1}}^{z_{l}} \frac{m(\xi)}{\sqrt{(\zeta-\xi)^{2}+r^{2}}} d \xi=m_{i} \ln \left[\frac{\zeta-z_{i-1}+\sqrt{\left(\zeta-z_{l-1}\right)^{2}+r^{2}}}{\zeta-z_{i}+\sqrt{\left(\zeta-z_{i}\right)^{2}+r^{2}}}\right] .
$$

For the case when $r=0$, we find

$$
\int_{z_{l-1}}^{z_{l}} \frac{m(\xi)}{\sqrt{(\zeta-\xi)^{2}+(0)^{2}}} d \xi=m_{l} \ln \left[\frac{z_{l}-\zeta}{z_{l-1}-\zeta}\right]
$$

where we are assuming $z_{k}>\zeta$, or else breakthrough would have occurred, and the model would be invalid anyway. To obtain the numerical solution, we also require the small-parameter approximation to first order, to get anywhere near the accuracy of previous results. The derivation and results used are as in Lucas [12].

Figure 7 shows the interface shapes produced for various values of $F^{\prime}$, with a 100-interval discontinuous mass flow approximation, and Table 4 summarises some of the details of the results. We can see that interface heights are less than for previous results, but it must be remembered that we are dealing with a different dimensionless parameter. Also, this program is significantly slower than previous models; a 50 -point spline took up to thirty minutes per iteration, indicating this program is about six times slower than our previous one.

\section{Conclusions}

A boundary-integral method has been developed which predicts the steadystate height of the interface between oil and water in an oil reservoir under the influence of an oil well pumping out oil to the surface. The models of 


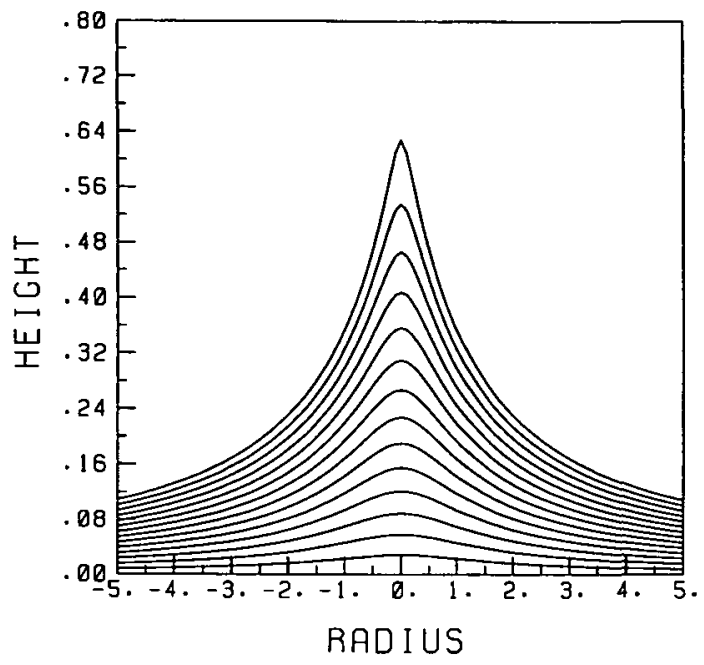

FIGURE 7. Interface shape for $F^{\prime}=0.2,0.4, \ldots, 2.8,50 \mathrm{pt}$ spline over range $0 \rightarrow 5$.

TABLE 4. Data from the constant downhole pressure model.

\begin{tabular}{|c|c|c|}
\hline$F^{\prime}$ & $\zeta(0)$ & Iterations \\
\hline 0.2 & 0.0288 & 2 \\
0.4 & 0.0585 & 2 \\
0.6 & 0.0850 & 3 \\
0.8 & 0.1217 & 3 \\
1.0 & 0.1554 & 3 \\
1.2 & 0.1908 & 3 \\
1.4 & 0.2282 & 4 \\
1.6 & 0.2680 & 4 \\
1.8 & 0.3107 & 4 \\
2.0 & 0.3570 & 5 \\
2.2 & 0.4084 & 5 \\
2.4 & 0.4666 & 6 \\
2.6 & 0.5360 & 7 \\
2.8 & 0.6281 & 9 \\
\hline
\end{tabular}

a point sink, a point source, a line sink of constant mass flow, and a line sink with variable mass flow to simulate a constant downhole pressure on a cylinder representing the actual well, have been used. While the point sink gave the most straightforward model for a numerical solution and discussion 
on critical flow rates, it can be seen that the more complicated constantdownhole-pressure model is a more realistic representation of the problem.

It must be emphasised that this is far from a complete model of the oilreservoir water-coning problem. We have only dealt with the steady-state problem, and so the important problem of the time taken for breakthrough to occur when pumping in excess of the critical time has not been addressed. In addition, the basic assumptions for the model are a major simplification. Of most importance is the assumption of a homogeneous medium of constant permeability $k$. In reality, the vertical permeability is roughly $1 / 10$ to $1 / 100$ of the horizontal permeability. This can be resolved by a simple stretching of the vertical coordinates, leading to a geometry with a constant $k$. Our major problem is that oil-bearing rock is rarely homogeneous, and is not of infinite size. Detailed knowledge of a particular rock layer's characteristics would be required for a more exact answer, which is often not feasible in a practical situation.

\section{Acknowledgments}

Acknowledgment is made of research support from A.R.C. This research was carried during the period when the three authors were in the Department of Mathematics at the University of Wollongong.

\section{References}

[1] M. Abramowitz and I. A. Stegun, (1972). Handbook of mathematical functions, (Dover, N. Y. 1965).

[2] J. Bear, (1972). Dynamics of Fluids in Porous Media, (McGraw-Hill, N. Y. 1972).

[3] J. Bear and G. Dagan, "Some exact solutions of interface problems by means of the hodograph method", J. Geophys. Res. 69 (1964) 1563-1572.

[4] J. R. Blake and A. Kucera, "Coning in oil reservoirs", Math. Scientist 13 (1988) 36-47.

[5] J. R. Blake, B. Taib and G. Doherty, "Transient Cavities near Boundaries Part 1. Rigid Boundary", J. Fluıd Mech. 170 (1986) 479-497.

[6] 1. L. Collings, "Two infinite-Froude number cusped free-surface flows due to a submerged line source or sink" J. Austral. Math. Soc. B28 (1987) 260-270.

[7] A. Craya, "Theoretical research on the flow of a non-homogeneous fluids" Homille Blanche 4 (1949) 44-55.

[8] R. E. Ewing, The Mathematics of Reservoir Simulation (SIAM, Philadelphia, 1983).

[9] E. J. Hinch, “The recovery of oil from underground reservoirs", PCH 6 (1985) 601-622.

[10] G. C. Hocking, "Cusp-like free-surface flows due to a submerged source or sink in the presence of a flat or sloping bottom” J. Austral. Math. Soc. B26 (1984) 470-486.

[11] G. C. Hocking, "Infinite Fronde number solutions to the problem of a submerged source or sink" J. Austral. Math. Soc. B29 (1988) 401-409.

[12] S. K. Lucas, "Water coning in oil reservoirs", (Univ. of Wollongong, Dep. Math., Honours Thesis 1988). 
[13] R. C. MacDonald, "Methods for numerical simulation of water and gas coning", Trans. Soc. Pet. Eng. 249 (1970) 423-434.

[14] M. Muskat, Physical principles of oil production, (McGraw-Hill, N. Y. 1949).

[15] R. Piessens, E. De Doncker-Kapenga, C. W. Uberhuber and D. K. Kahauer, QUADPACK, A subroutine package for automatic integration, (Springer-Verlag, Berlin, 1983).

[16] E. O. Tuck and J. Vanden-Broeck, "A cusp-like free-surface flow due to a submerged source or sink" J. Austral. Math. Soc. B25 (1984) 443-450. 